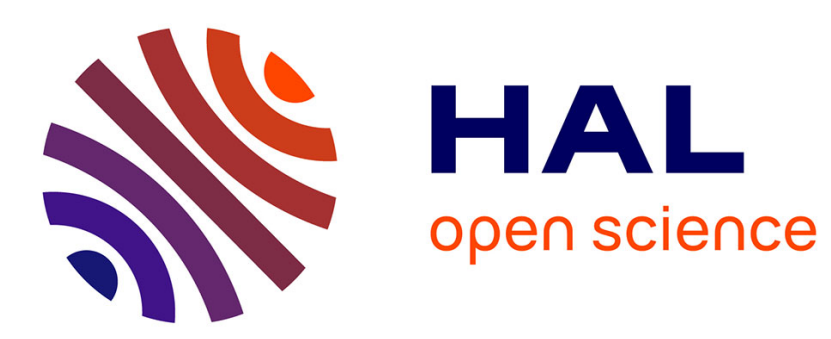

\title{
On the optimization of resources for short frame synchronization
}

Alex The Phuong Nguyen, Frédéric Guilloud, Raphaël Le Bidan

\section{To cite this version:}

Alex The Phuong Nguyen, Frédéric Guilloud, Raphaël Le Bidan. On the optimization of resources for short frame synchronization. Annals of Telecommunications - annales des télécommunications, 2020, 10.1007/s12243-020-00787-y . hal-02914757

\section{HAL Id: hal-02914757 https://imt-atlantique.hal.science/hal-02914757}

Submitted on 15 Feb 2022

HAL is a multi-disciplinary open access archive for the deposit and dissemination of scientific research documents, whether they are published or not. The documents may come from teaching and research institutions in France or abroad, or from public or private research centers.
L'archive ouverte pluridisciplinaire HAL, est destinée au dépôt et à la diffusion de documents scientifiques de niveau recherche, publiés ou non, émanant des établissements d'enseignement et de recherche français ou étrangers, des laboratoires publics ou privés. 


\title{
On the Optimization of Resources for Short Frame Synchronization
}

\author{
Alex The Phuong Nguyen • Frédéric \\ Guilloud • Raphaël Le Bidan
}

Received: date / Accepted: date

\begin{abstract}
We consider the transmissions of successive short packets. Each of them combines information to be transmitted (codeword) with information for synchronizing the frame (syncword). For short packets, the cost of including syncwords is no longer negligible and its design requires careful optimization. In particular, a trade-off arises depending on the way the total transmit power or the total frame length is split among the syncword and the codeword. Assuming optimal finite-length codes, we develop tight upper bounds on the probability of erroneous synchronization, for both frames with concatenated syncword and frames with superimposed syncword. We use these bounds to optimize this trade-off. Simulation results show that the proposed bounds and analysis have practical relevance for short packet communication system design.
\end{abstract}

Keywords Frame synchronization - short-packet transmission · finite blocklength $\cdot$ mMTC $\cdot$ URLLC

\section{Introduction}

Modern digital communication systems transmit data in packets or frames where the data of interest is incorporated together with additional control

\footnotetext{
Alex The Phuong Nguyen

E-mail: thephuong.nguyen@imt-atlantique.fr

IMT Atlantique, Lab-STICC, 29238 Brest, France

Frédéric Guilloud

E-mail: frederic.guilloud@imt-atlantique.fr

Tel.: +123-45-678910

IMT Atlantique, Lab-STICC, 29238 Brest, France

Raphaël Le Bidan

E-mail: raphael.lebidan@imt-atlantique.fr

Tel.: +123-45-678910

IMT Atlantique, Lab-STICC, 29238 Brest, France
} 
information referred to as meta-data. Specifically, at the PHY layer, two important types of meta-data are pilots symbols (e.g. for channel estimation) and frame synchronization (FS) sequences. For large packet size, the cost of inserting these meta-data is negligible. However, considering a short and fixed packet length, increasing the meta-data length reduces both the spectral efficiency and the coding length. Conversely, shortening the length of meta-data degrades the performance of their intended usages (e.g worse channel estimation or frame synchronization). Hence, a trade-off between meta-data length and code length is to be found for short packets. With the objective of maximizing the throughput, the authors of [1] demonstrate this trade-off for pilotsymbol based channel estimation. In this paper, we are interested in non-blind frame synchronization for short-packet transmissions.

The typical FS strategy is to estimate the position of a known symbol sequence (synchronization word, SW) within the frames. There are two standard techniques to detect such a known sequence. The first one is based on binary hypothesis testing and is often used for burst (one-shot) transmissions. In [2], the authors derive optimum (minimizing error probability) metrics for binary signaling (SW and data) in Additive White Gaussian Noise (AWGN) channels. Recently, studies about the overhead of one-shot FS in AWGN channels has been conducted in [3].

The second technique assumes that frames are transmitted continuously and successively, and consists in maximizing a metric over the period of a frame. Although binary hypothesis testing is still possible, one can obtain better performance by exhaustive search within the frame duration.

In both techniques, the channel model and the data distribution determine the metric to be used. For example, for AWGN channels with binary modulation, the optimum metric minimizing FS error was provided in [4]. As an extension of [4], the authors of [5] present ML metrics for general M-ary phase-coherent and phase-noncoherent SW in AWGN. For Rayleigh fading model, one can find in [6] the optimum metrics for non-coherent detection. In the previous papers, no analytical synchronization error rate analysis are carried out except in [7] where the authors provide both metrics and analytical performance results in the particular case of binary modulation over AWGN.

In this paper, we propose an optimization of the ressources allocated to the overhead and coded data, respectively, by using the finite-length channel decoding performance of [8]. To this aim, we provide an analysis of FS error with the assumption of continuous transmission and known frame-length, with periodically-embedded SW. This setup is frequently encountered e.g. in dense wireless networks with many users, or in broadcast channels where a receiver may connect anytime. This connection must go through a synchronization process that includes FS. Note that once FS is established, the receiver can simply ignore the subsequent SW and, therefore, there is no need to synchronize the frame on each received packet. Our contribution is threefold. Assuming optimal finite-length channel codes, we first derive approximations on the probability of false FS for the correlation metric, for two different structures of frame: concatenated SW (CSW) and superimposed SW (SSW). Then these approxi- 
mations are used to optimize the total power distribution among the SW and the codeword that minimizes the overall frame error probability (FEP). Finally, the relevance of the proposed optimization is validated by confronting the theoretical predictions to the simulation results of a practical modulation and coding setup. Note that a related problem has been considered in [3], albeit in a different setting (burst transmissions), and with a different approach (hypothesis testing). Moreover, [3] assumes a specific SW whereas the present results apply to a much broader SW class. The present work and [3] are thus complementary.

The paper is organized as follows. Section 2 describes the system model and problem formulation. The analysis of false synchronization probability is carried out in Section 3. Numerical evaluation and comparison between the two frame structures follow in Section 4.

Notation. Scalar random variables are denoted by upper case letters e.g. $X$, whereas their realizations are written in lower case e.g. $x$. Boldface upper case letters e.g. $\mathbf{X}$ signify random vectors and boldface lower case letters e.g. $\mathbf{x}$ are used for their realizations. Notations $\mathbf{x}_{\mathcal{F}(n)}, \mathbf{x}_{\mathcal{L}(n)}$ and $\mathbf{x}_{i:(n)}$ mean the first $n$ elements, the last $n$ elements and the $n$ elements from the position $i$ of $\mathbf{x}$. By $X \stackrel{d}{=} Y$ we mean that two random variables $X$ and $Y$ have the same distribution.

\section{System Model and Problem Statement}

\subsection{Frame Structures}

We consider continuous transmissions of successive frames $\mathbf{X}$ of length $N$. This frame is built using a fixed SW $\mathbf{s} \in \mathbb{C}^{m}$ and a random codeword $\mathbf{C} \in \mathbb{C}^{n}$ which carries the coded data. There are two models for incorporating $\mathbf{s}$ and $\mathbf{C}$ in $\mathbf{X}$. The first one is by concatenation (CSW), as illustrated in Fig. 1-(a): $\mathbf{X}=[\mathbf{s} ; \mathbf{C}]$ and $N=m+n$. Note that similar to [7], we assume $m<N / 2$. The second one is by superposition (SSW), as illustrated in Fig. 1-(b): $\mathbf{X}=\mathbf{s}+\mathbf{C}$ and $m=n=N$.

The SW has power $\|\mathbf{s}\|^{2}=m \rho_{s}$ where $\rho_{s}$ denotes the average power per SW symbol. Codewords $\mathbf{C}$ have rate $R=k / n$ bits/symbol and constant power $\|\mathbf{C}\|^{2}=n \rho_{c}$ with $\rho_{c}$ being the average power per code symbol. The average power of a transmitted frame is thus $\|\mathbf{X}\|^{2}=N \rho_{t}=m \rho_{s}+n \rho_{c}$. We further assume that the codewords are uniformly distributed on the complex hypersphere of radius $\sqrt{n \rho_{c}}$ (shell codes). This assumption follows from Shannon's achievability proof of the AWGN channel capacity theorem establishing that optimal codes for complex AWGN channels in the asymptotic regime consist of dense packing of signal points within a sphere of $\mathbb{C}^{n}$. It is worth mentioning that, to date, the distribution of optimal finite-length codes on the AWGN channel remains unknown. Moreover, the use of shell codes has several advantages including not only tractable analysis or consistency with asymptotic 
(a)

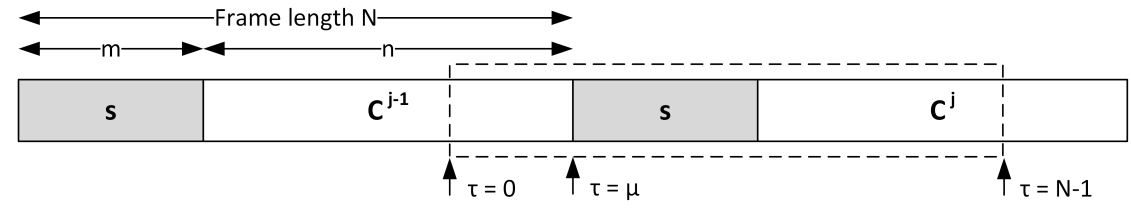

(b)

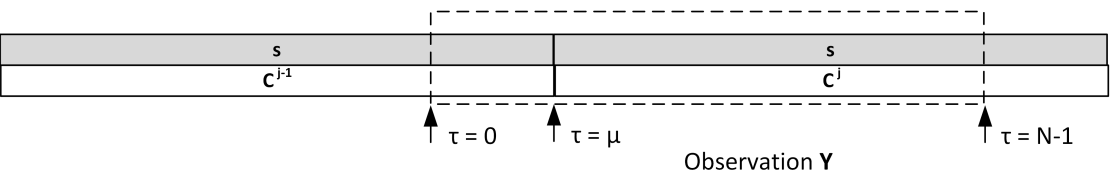

Fig. 1: Frame structure of the $(j-1)$-th frame and the $j$-th frame for (a) concatenated SW (CSW), and (b) superimposed SW (SSW). The received observation is buffered in vector $\mathbf{Y}$ where the frame begins at relative position $\tau=\mu$.

capacity results as the code length increases, but also the possibility to compare to other similar or related finite-length analysis reported in the literature, most of them relying on similar assumptions, see e.g. [3, 8, 9].

At the receiver side, the observation is buffered in a length- $N$ vector $\mathbf{Y}$. Let $0 \leq \mu<N$ denote the start frame index within $\mathbf{Y}$, as illustrated in Fig. 1: $\mathbf{Y}$ contains the last $\mu$ symbols of previous frame $\mathbf{X}^{j-1}$ followed by the first $N-\mu$ symbols of current frame $\mathbf{X}^{j}: \mathbf{Y}=\left[\mathbf{X}_{\mathcal{L}(\mu)}^{j-1} ; \mathbf{X}_{\mathcal{F}(N-\mu)}^{j}\right]+\mathbf{W}$ with $\mathbf{W} \sim$ $\mathcal{C N}\left(\mathbf{0}, \mathbf{I}_{N}\right)$. Since the additive noise has unit variance per complex coordinate, $\rho_{t}$ can be thought as receive signal-to-noise ratio (SNR).

\subsection{Problem Statement}

Neglecting the probability that channel decoding may succeed in the case of incorrect synchronization ${ }^{1}$, an upper bound $P_{\mathrm{E}}$ on the FEP after decoding reads:

$$
P_{\mathrm{E}}=1-\left(1-P_{f}\left(m, \rho_{s}, n, \rho_{c}\right)\right)\left(1-P_{d}\left(n, R, \rho_{c}\right)\right),
$$

where $P_{f}\left(m, \rho_{s}, n, \rho_{c}\right)$ denotes the probability of false synchronization and $P_{d}\left(n, R, \rho_{c}\right)$ denotes the probability of decoding error conditioned to a successful synchronization for a code of rate $R$ and length $n$ at $\operatorname{SNR} \rho_{c}$.

We operate at fixed frame size $N$ and fixed total transmit power $\|\mathbf{X}\|^{2}$, and we would like $P_{\mathrm{E}}$ to be as small as possible. For both CSW and SSW, a fundamental trade-off arises between decoding and synchronization which can be characterized by:

$$
\hat{\beta}=\underset{0 \leq \beta \leq 1}{\arg \min } P_{\mathrm{E}}
$$

\footnotetext{
${ }^{1}$ Note that for most practical codes and decoders the probability of correct decoding in case of a synchronization error is very small. Hence, $P_{\mathrm{E}}$ as defined in (1) is expected to be a tight upper bound of the FEP.
} 
where $\beta \triangleq\|\mathbf{s}\|^{2} /\|\mathbf{X}\|^{2}$. Solving (2) requires the analytic expressions of $P_{f}$ and $P_{d}$. For the latter we resort to the RCU bound [9] which is a tight upper bound on the decoding probability achievable by a suitable pair of AWGN finite-length channel code and decoder. The evaluation of $P_{f}$ is the subject of next Section.

\subsection{Upper bound on false synchronization probability}

Given a received vector $\mathbf{y}$, the probability of false synchronization $P_{f}\left(m, \rho_{s}, n, \rho_{c}\right)$ is the probability $\operatorname{Pr}\{\hat{\mu} \neq \mu\}$ where $\hat{\mu}=\underset{0 \leq \tau<N}{\operatorname{argmax}} f(\mathbf{y}, \tau)$ is the estimation of frame beginning index, and $f(\mathbf{y}, \tau)$ is the considered metric that is evaluated for each possible SW location $0 \leq \tau<N$. False synchronization probability is thus defined as

$$
P_{f}=\operatorname{Pr}\{\hat{\mu} \neq \mu\}=\operatorname{Pr}\left\{\bigcup_{\tau \neq \mu}[f(\mathbf{Y}, \mu) \leq f(\mathbf{Y}, \tau)]\right\}
$$

The exact calculation of $P_{f}$ is usually hard since the events $[f(\mathbf{Y}, \mu) \leq f(\mathbf{Y}, \tau)]$ are generally not disjoint. For that reason, we resort to the union bound and upper-bound $P_{f}$ by

$$
P_{f} \leq P_{f, u} \triangleq \sum_{\tau \neq \mu} \mathcal{P}_{e}(\tau) \text { where } \mathcal{P}_{e}(\tau) \triangleq \operatorname{Pr}\{f(\mathbf{Y}, \mu) \leq f(\mathbf{Y}, \tau)\}
$$

Therefore, $P_{\mathrm{E}}$ is upper bounded by $P_{\mathrm{E}, \mathrm{u}}$

$$
P_{\mathrm{E}, \mathrm{u}}=1-\left(1-P_{f, u}\right)\left(1-P_{d}\right)
$$

Our objective is then to search for the value $\beta$ that minimizes (2), but wherein $P_{\mathrm{E}}$ has been replaced by $P_{\mathrm{E}, \mathrm{u}}$. We are now left with the central problem of evaluating the pairwise error probability $\mathcal{P}_{e}(\tau)$.

\section{Pairwise Synchronization Error Probability}

A common engineering practice for FS is to look for the position that maximizes the correlation metric. In this section, we derive the pairwise probability expressions given in (4) first for the coherent, then for the non-coherent receiver. Note that an ML based metric derivation can be found in [10]. 
3.1 Correlation Metric for the Coherent Receiver

Assuming a coherent receiver with perfect phase offset correction, we obtain [11, Ch.3, p.69]:

$$
\hat{\mu}=\underset{0 \leq \tau<N}{\operatorname{argmax}} \operatorname{Re}\left\{\mathbf{s}^{H} \mathbf{y}_{\tau:(m)}\right\} \triangleq \underset{0 \leq \tau<N}{\operatorname{argmax}} f_{\mathrm{C}}(\mathbf{y}, \tau)
$$

Here circular indexing within the receive buffer is assumed in the computation of the scalar product $\mathbf{s}^{H} \mathbf{y}_{\tau:(m)}$.

\subsubsection{Concatenation $S W(C S W)$}

Evaluating $\mathcal{P}_{e}(\tau)$ for $f_{\mathrm{C}}(\mathbf{y}, \tau)$ requires characterizing the probability distribution of $f_{\mathrm{C}}(\mathbf{Y}, \mu)$ and $f_{\mathrm{C}}(\mathbf{Y}, \tau)$ for $\tau \neq \mu$. Two cases have to be distinguished depending on whether the computation of these two metrics overlap. For $|\tau-\mu| \geq m$ and provided $m<N / 2$, the random variables $f_{\mathrm{C}}(\mathbf{Y}, \mu)$ and $f_{\mathrm{C}}(\mathbf{Y}, \tau)$ are computed from distinct received symbols, thus independent. On the other hand, for $|\tau-\mu|<m$, the two random variables overlap on $m-|\tau-\mu|$ coordinates and the independence assumption no longer holds. Let $\mathcal{P}_{e, \text { up }}(\tau) \triangleq \mathcal{P}_{e}(\tau)$ when $|\tau-\mu| \geq m, \mathcal{P}_{e, \text { lo } 1}(\tau) \triangleq \mathcal{P}_{e}(\tau)$ when $0<\tau-\mu<m$ and $\mathcal{P}_{e, \operatorname{lo} 2}(\tau) \triangleq \mathcal{P}_{e}(\tau)$ when $-m<\tau-\mu<0$,

$$
P_{f, u}=(N-2 m+1) \mathcal{P}_{e, \mathrm{up}}(\tau)+\sum_{0<\tau-\mu<m} \mathcal{P}_{e, \mathrm{lo} 1}(\tau)+\sum_{-m<\tau-\mu<0} \mathcal{P}_{e, \mathrm{lo} 2}(\tau)
$$

The exact and approximate derivation of $\mathcal{P}_{e, \text { up }}(\tau), \mathcal{P}_{e, \text { lo } 1}(\tau)$ and $\mathcal{P}_{e, \text { lo2 }}(\tau)$ can be found in [10]. Note that the proposed approximation is quite accurate and simple to manipulate. Denoting by $\mathcal{Q}(x)=\frac{1}{\sqrt{2 \pi}} \int_{x}^{+\infty} e^{-\frac{u^{2}}{2}} d u$ the tail distribution function of the standard Normal distribution, this approximation reads:

$$
\mathcal{P}_{e, \mathrm{up}}(\tau) \approx \mathcal{Q}\left(\frac{\|\mathbf{s}\|}{\sqrt{1+\rho_{c} \frac{n}{2 n-3}}}\right), \mathcal{P}_{e, \mathrm{lo} 1}(\tau) \approx \mathcal{Q}\left(\frac{\nu_{1}}{\sigma_{1}}\right), \mathcal{P}_{e, \mathrm{lo} 2}(\tau) \approx \mathcal{Q}\left(\frac{\nu_{2}}{\sigma_{2}}\right)
$$

with $\nu_{1}=\|\mathbf{s}\|^{2}-\operatorname{Re}\left\{\mathbf{s}_{\mathcal{F}(m-\tau+\mu)}^{H} \mathbf{s}_{\mathcal{L}(m-\tau+\mu)}\right\}$ and $\sigma_{1}^{2}=\nu_{1}+\left\|\mathbf{s}_{\mathcal{L}(\tau-\mu)}\right\|^{2} \rho_{c} \frac{n}{2 n-3}$ and $\nu_{2}=\|\mathbf{s}\|^{2}-\operatorname{Re}\left\{\mathbf{s}_{\mathcal{L}(m-\mu+\tau)}^{H} \mathbf{s}_{\mathcal{F}(m-\mu+\tau)}\right\}$ and $\sigma_{2}^{2}=\nu_{2}+\left\|\mathbf{s}_{\mathcal{F}(\mu-\tau)}\right\|^{2} \rho_{c} \frac{n}{2 n-3}$. A comparison between the numerical evaluation of the union bound $P_{f, u}$, its approximation using (8) and Monte-Carlo simulations is provided in Fig. 2. The gap between the approximations and the numerical evaluation of the union bound is only distinguishable for important overheads and small frame lengths $N$.

\subsubsection{Superposition $S W(S S W)$}

In the case of a superimposed SW, the pairwise probability is approximated as $[12]$

$$
\mathcal{P}_{e}(\tau)=\operatorname{Pr}\left\{f_{C}(\mathbf{Y}, \mu) \leq f_{C}(\mathbf{Y}, \tau)\right\} \approx \mathcal{Q}\left(\nu_{3} / \sigma_{3}\right)
$$

where $\nu_{3}=\|\mathbf{s}\|^{2}-\operatorname{Re}\left\{\mathbf{s}_{\tau:(m)}^{H} \mathbf{s}_{\mu:(m)}\right\}$ and $\sigma_{3}^{2}=\frac{1+\rho_{c}}{2}\left\|\mathbf{s}_{\mu:(m)}-\mathbf{s}_{\tau:(m)}\right\|^{2}$. 


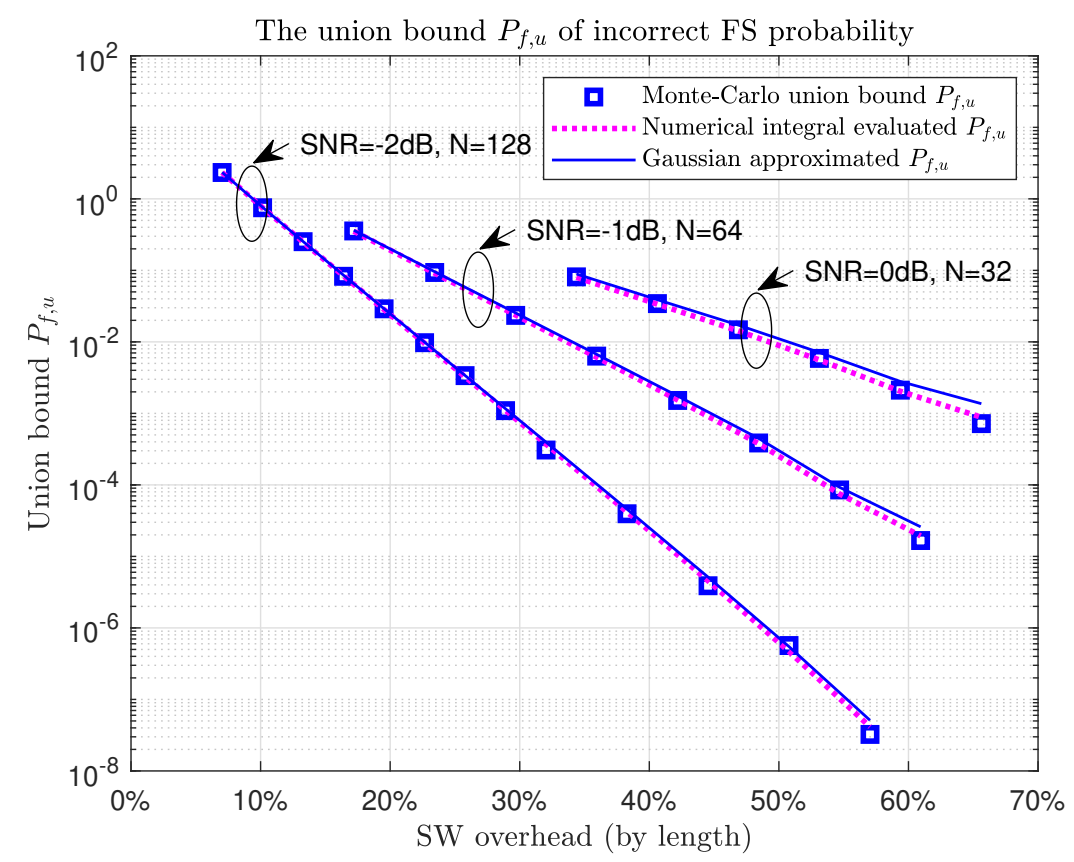

Fig. 2: The union bound $P_{f, u}$ of incorrect FS probability vs. SW overhead $\beta=\|\mathbf{s}\|^{2} /\|\mathbf{X}\|^{2}$ at uniform power $\rho_{s}=\rho_{c}$ for several SNR $\rho_{t}$ and frame lengths $N$.

\subsection{Correlation Metric for the Non-Coherent Receiver}

We note that the (real-valued) correlation rule (6) is the consequence of the assumption of having a coherent receiver, i.e. with perfect knowledge and compensation of any phase offset that may arise during the transmission. In such a context, (6) is the optimal correlation rule obtained from MaximumLikehood principles (see e.g. [11, Ch.3, P.69]). In the presence of a random phase offset (non-coherent setting), another form of correlation rule arises, based this time upon the absolute value or the $l^{2}$ norm [11, Ch.3, P.70]:

$$
\hat{\tau}=\underset{0 \leq \tau<N}{\operatorname{argmax}}\left|\mathbf{s}^{H} \mathbf{y}_{\tau:(m)}\right|^{2} \triangleq \underset{0 \leq \tau<N}{\operatorname{argmax}} f_{\mathrm{A}}(\mathbf{y}, \tau)
$$

In this section, we focus on the CSW structure, the SSW structure being left as a perspective for future work. We thus resort to the upper-bound approximation:

$$
P_{f, u} \approx(N-1) \mathcal{P}_{e, \mathrm{up}}(\tau)
$$

by assuming that the SW is properly-designed in the sense that it mimics random coded data. This assumption is all the more justified that the ratio $m / N$ is small [7] and that $\mathbf{s}$ and $\mathbf{C}$ use the same modulation alphabet. 
Define $\mathcal{P}_{e, \text { up }}(\tau) \triangleq \operatorname{Pr}\left\{f_{A}(\mathbf{Y}, \mu) \leq f_{A}(\mathbf{Y}, \tau)\right\}$ for $|\tau-\mu| \geq m$ hence $f_{A}(\mathbf{Y}, \mu)$ and $f_{A}(\mathbf{Y}, \tau)$ are independent. We have $\mathbf{s}^{H}\left(\mathbf{s}+\mathbf{W}_{\tau:(m)}\right) \sim \mathcal{C N}\left(\|\mathbf{s}\|^{2},\|\mathbf{s}\|^{2}\right)$, from which we obtain $\frac{2}{\|\mathbf{s}\|^{2}} f_{A}(\mathbf{Y}, \mu)=\frac{2}{\|\mathbf{s}\|^{2}}\left|\mathbf{s}^{H}\left(\mathbf{s}+\mathbf{W}_{\tau:(m)}\right)\right|^{2} \sim \chi_{2}^{2}\left(2\|\mathbf{s}\|^{2}\right)$. Similarly, $f_{A}(\mathbf{Y}, \tau)=\left|\mathbf{s}^{H}\left(\mathbf{C}_{|\tau-\mu|:(m)}+\mathbf{W}_{\tau:(m)}\right)\right|^{2} \stackrel{d}{=}\left|\|\mathbf{s}\|\left(\mathbf{C}_{|\tau-\mu|:(1)}+\mathbf{W}_{\tau:(1)}\right)\right|^{2}$ then $\frac{2}{\|\mathbf{s}\|^{2}}\left(1+\rho_{c}\right)^{-1} f_{A}(\mathbf{Y}, \tau) \sim \chi_{2}^{2}(0)$ where we have used the symmetric isotropic property and the property of point-wise convergence of a codeword $\mathbf{C}$ to $\mathcal{C N}\left(\mathbf{0}, \rho_{c} \mathbf{I}_{n}\right)$ [13, Sections 3.1 and 7.2].

By combining these results, $\mathcal{P}_{e, \text { up }}(\tau)=\operatorname{Pr}\left\{\frac{f_{A}(\mathbf{Y}, \mu)}{f_{A}(\mathbf{Y}, \tau)}<1\right\} \approx F_{U}\left(1+\rho_{c}\right)$ where $U \triangleq \chi_{2}^{2}\left(2\|\mathbf{s}\|^{2}\right) / \chi_{2}^{2}(0)$ is a singly non-central F-distributed random variable.

\section{Numerical evaluation}

\subsection{Coherent versus Non-coherent}

Figure 3 compares the frame error rate obtained using the non-coherent correlation rule (10) to the coherent rule (6) for a frame of $N=256$ symbols, $k=\lceil N / 3\rceil=86$ information bits. The average power for the SW and codeword symbols is the same and equal to the SNR: $\rho_{c}=\rho_{s}=\rho_{t}=-2 \mathrm{~dB}$ for the concatenated SW structure. The results obtained with the non-coherent correlation metric are worse than those for the coherent rule at small overhead $(<25 \%)$. However, the corresponding receiver is less complex and undoubtedly more robust. Note that in both cases the frame error rate estimated by the Monte-Carlo simulation comes very close to the proposed frame error rate approximation.

\subsection{Concatenation versus Superposition SW}

In Figure 4, the proposed approximations on frame error rate as a function of the SW overhead are compared to the Monte-Carlo simulation results of $P_{E}$ for a frame of length $N=129$ incorporating $k=65$ information bits. The two SW overhead structures are compared: concatenation and superimposition. These comparisons are performed for three different SNR. Compared to the Monte-Carlo simulations, the theoretical approximations are again very close. We also note that the best overhead trade-off for each frame structure yields equivalent error rates so that no best solution arrises here.

\subsection{Theoretical versus Practical Performance}

The proposed optimization strategy assumes optimal finite-length AWGN codes whose performance are upper bounded by the RCU [9]. It is the object 


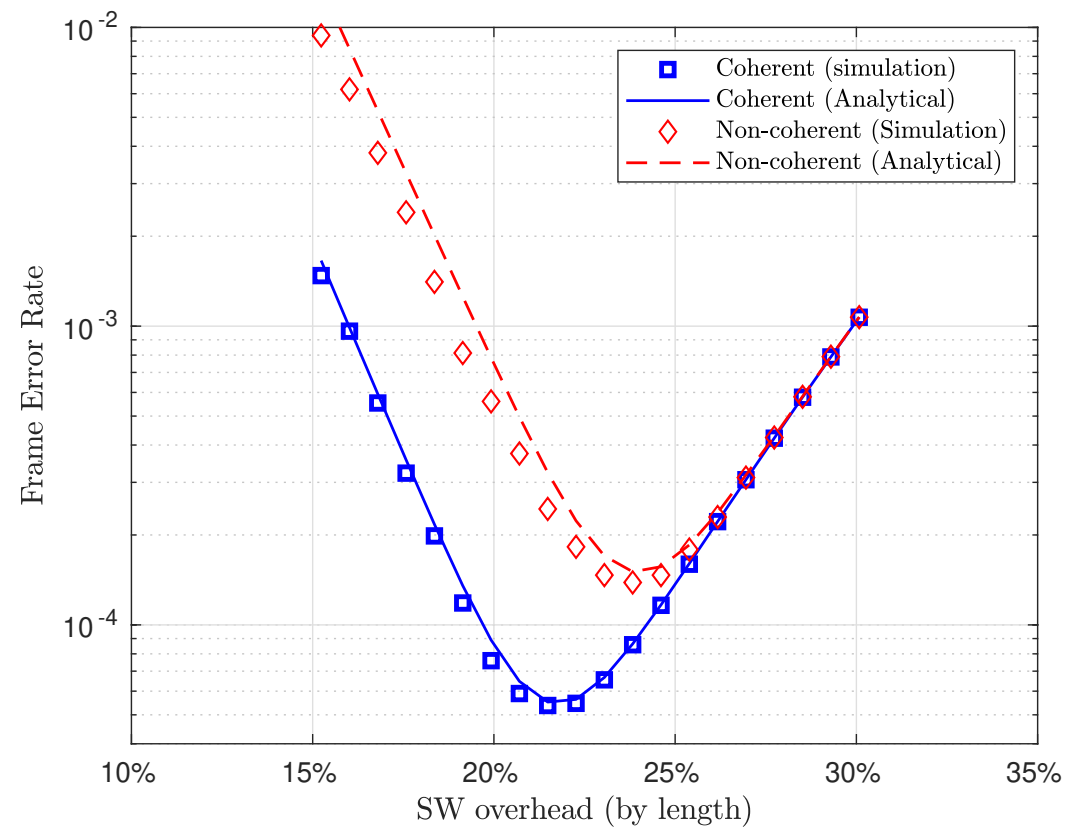

Fig. 3: Coherent versus non-coherent correlation detection comparison for CSW. Frame length $N=256$ for $k=86$ info bits and SNR $=-2 \mathrm{~dB}$.

of this subsection to check whether the trade-offs achieved using our theoretical performance approximations is the same as those that could be obtained with more practical coding schemes. Hereafter, we consider a practical QPSK transmission scheme with the 5G Polar code and its 24-bit CRC [14] companion leading to the total length $n=2 \times(256-m)$ bits and dimension $k=86$. The frame detection is performed using the coherent correlation metric. The 5G Polar code will be decoded by the CRC-aided Successive Cancellation List (CA-SCL) decoder with list sizes $L=32$ and $L=256$. Note that the larger the list the closer to ML performance the CA-SCL decoder is, but at the cost of increased computational complexity.

The results are depicted in Fig. 5 for two different concatenated SW optimization strategies: (a) varying the SW length while keeping a constant uniform transmit power (on the left part), and (b) varying the SW-over-data power ratio while keeping a constant SW length $m=55$. In both cases the frame length is $N=256$ and the SNR is $\rho_{t}=-2 \mathrm{~dB}$. The blue curves correspond to the theoretical approximations and the square blue marks correspond to the Monte-Carlo estimations. The light blue and black colors correspond to the performance of the practical scheme. We observe that the trade-offs obtained with our proposed theoretical approximations match those of the 


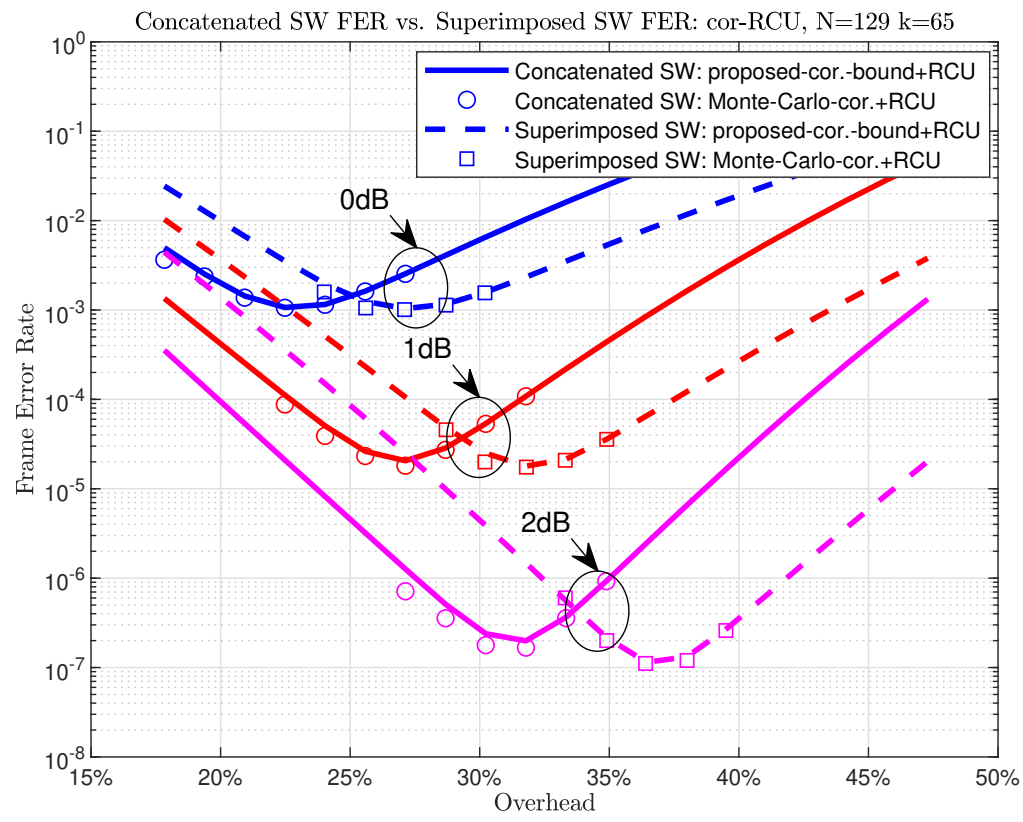

Fig. 4: Comparison of $P_{\mathrm{E}, \mathrm{u}}$ between concatenated SW (assuming $\rho_{s}=\rho_{c}=\rho_{t}$ ) and superimposed SW. Frame length $N=129$ transporting $k=65$ bits for several SNR $\rho_{t}$. The theoretical approximations are on $P_{\mathrm{E}, \mathrm{u}}$ and the MonteCarlo performance are on $P_{\mathrm{E}}$.

practical scheme simulations $(\approx 22 \%)$. The matching is all the more evident as $L$ is increased.

Note that a frame error rate gap is observed between the theoretical performance and the practical scheme performance. This gap is due both to code imperfectness and sub-optimal decoding. The latter issue can be addressed with a larger list size in the CA-SCL decoder.

\section{Conclusion}

In this paper, we have considered upper bounds on the false synchronization probability in the context of continuous transmissions over AWGN channel. A synchronization word is assumed to be either concatenated or superimposed to the data symbols. Simple approximations of the proposed bounds have been derived and compared to Monte-Carlo simulations showing that our results are tight. Then these theoretical approximations are used together with recent results on the finite blocklength coding performances in order to optimize the power or length overhead between the synchronization word and the data 


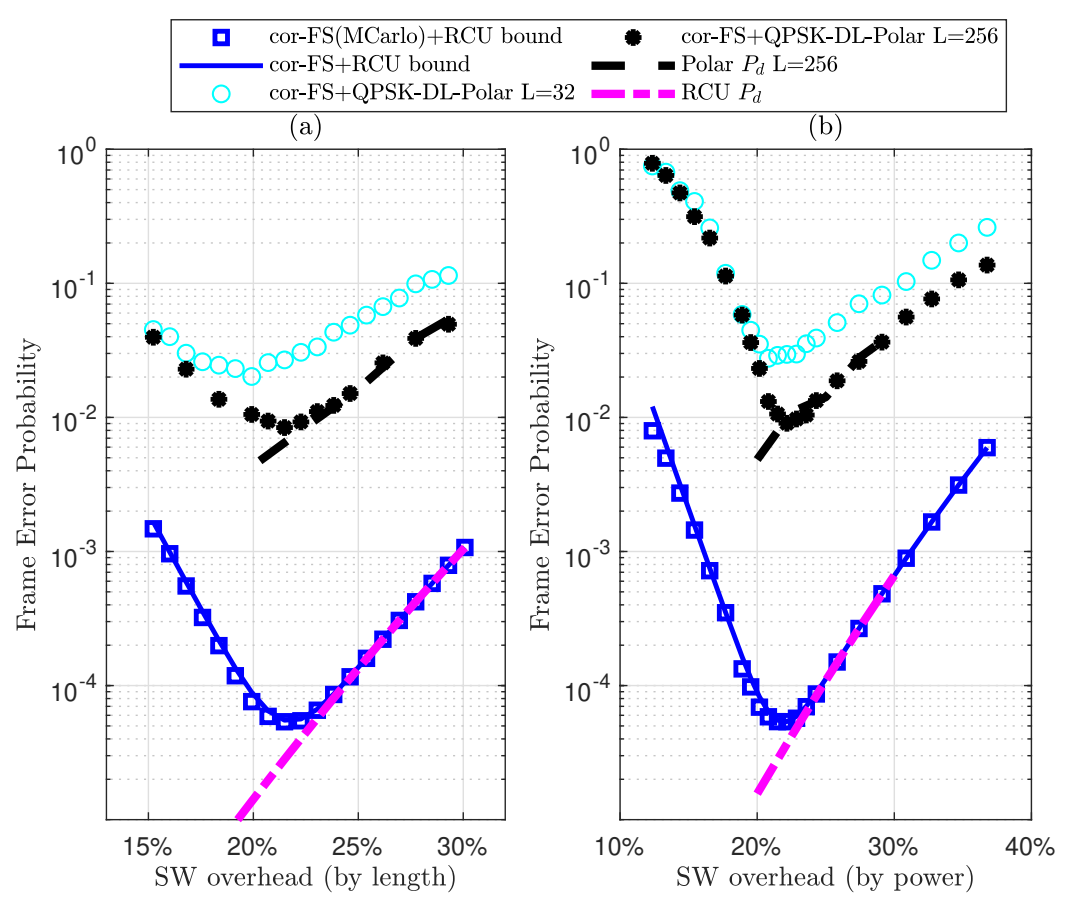

Fig. 5: CSW. $P_{\mathrm{E}}$ vs. SW overhead at frame length $N=256$ and SNR $\rho_{t}=-2 \mathrm{~dB}$ with (a) uniform transmit power $\rho_{s}=\rho_{c}$ but varying SW length $m$, or (b) fixed SW length $m=55$ but varying SW-codeword power ratio. The continuous blue curves are $P_{\mathrm{E}, \mathrm{u}}(5)$, the square curves are $P_{\mathrm{E}}(1)$ and the Polar-code curves are FEP.

symbols for a given total transmitted average symbol energy and a total frame length. The comparison between using a synchronization word superimposed or concatenated to the data symbols which shows that the performance are pretty much the same. Finally, these optimized theoretical overheads are shown to coincide with practical 3GPP 5G-NR Downlink Polar codes simulations with QPSK modulations.

\section{References}

1. Östman, J., Durisi, G., Ström, E.G., Coşkun, M.C., Liva, G.: Short packets over block-memoryless fading channels: Pilot-assisted or noncoherent transmission? IEEE Transactions on Communications 67(2), 1521-1536 (2019)

2. Chiani, M., Martini, M.G.: On sequential frame synchronization in AWGN channels. IEEE Transactions on Communications 54(2), 339-348 (2006)

3. Bana, A.S., Trillingsgaard, K.F., Popovski, P., de Carvalho, E.: Short packet structure for Ultra-Reliable Machine-type Communication: Tradeoff between detection and decoding. In: IEEE International Conference 
on Acoustics, Speech and Signal Proceeding (ICASSP), pp. 6608-6612. IEEE, Calgary, Alberta, Canada (2018)

4. Massey, J.: Optimum frame synchronization. IEEE Transactions on Communications 20(2), 115-119 (1972)

5. Lui, G., Tan, H.: Frame synchronization for Gaussian channels. IEEE Transactions on Communications 35(8), 818-829 (1987)

6. Jia, H., Dodds, D.E.: Frame synchronization for PSAM in AWGN and Rayleigh fading channels. In: Canadian Conference on Electrical and Computer Engineering (CCECE), pp. 44-50. IEEE, Windsor, Canada (2005)

7. Chiani, M., Martini, M.G.: Analysis of optimum frame synchronization based on periodically embedded sync words. IEEE Transactions on Communications 55(11), 2056-2060 (2007)

8. Erseghe, T.: Coding in the finite-blocklength regime: Bounds based on laplace integrals and their asymptotic approximations. IEEE Transactions on Information Theory 62(12), 6854-6883 (2016)

9. Polyanskiy, Y., Poor, H.V., Verdú, S.: Channel coding rate in the finite blocklength regime. IEEE Transactions on Information Theory 56(5), 2307-2359 (2010)

10. Nguyen, A.T.P., Le Bidan, R., Guilloud, F.: Trade-off between frame synchronization and channel decoding for short packets. IEEE Communications Letters (2019)

11. Ling, F.: Synchronization in digital communication systems. Cambridge University Press (2017)

12. Nguyen, A.T.P., Le Bidan, R., Guilloud, F.: Superimposed frame synchronization optimization for Finite block-length regime. IEEE Wireless Communications and Networking Conference (WCNC) Workshops (2019)

13. Zamir, R.: Lattice Coding for Signals and Networks. Cambridge University Press, New York, NY, USA (2014)

14. 3GPP 38 212: LTE; E-UTRA; 5G; NR; Multiplexing and channel coding Rel.15. 3GPP (2019). V15.2.0 